\title{
Design of Multi-standard Low Noise Amplifier based on Active Inductor
}

\author{
Weiyin Wang, Hailian Liang, Linna Zhao, Xiaofeng Gu* \\ Key Laboratory of Advanced Process Control for Light Industry (Ministry of Education) \\ Department of Electronic Engineering; Jiangnan University \\ Wuxi, China \\ xgu@jiangnan.edu.cn
}

\begin{abstract}
A current-controlled multi-standard low-noise amplifier (LNA) has been proposed based on the active inductor. The novel folded-cascode circuit topology with feedback resistance ensures good quality factor and linearity of the active inductor. The current-controlled inductance makes the LNA work well with various wireless communication standards such as TD-SCDMA, CDMA2000, WCDMA and Bluetooth. Simulation results indicate that the multi-standard LNA designed using $0.18 \mu \mathrm{m}$ CMOS technology has a high gain $(>11.6 \mathrm{~dB})$, low noise figure $(<1.851 \mathrm{~dB})$, good input impedance matching ( $<-54.01 \mathrm{~dB})$ and stable output impedance matching $(<-26.78 \mathrm{~dB})$.
\end{abstract}

Keywords-multi-standard; LNA; active inductor; currentcontrolled;

\section{INTRODUCTION}

With the rapid development of the $3 \mathrm{G}$ cellular system, it has become essential to integrate various wireless communication standards into a cell phone. Due to the increasing demand on global roaming and all-in-one cell phones, the interest in developing multi-standard transceivers has been increasing. Currently, the most popular standards in China include TD-SCDMA, CDMA2000, WCDMA and Bluetooth. Operating frequencies of these standards are listed in Table 1. Many researches have been done on multistandard low noise amplifier (LNA). Recently, the ultrawideband (3.1-10.6 GHz) LNA has also been designed [1], but it has a high noise figure (NF) and bad impedance matching for the $3 \mathrm{G}$ cellular system. In the dual-input pseudo-switch radio frequency (RF) LNA, RF switch introduces large noise when shifted among various matching circuits. In this work, we propose a novel multi-standard LNA based on the active inductor. The current-controlled inductance makes the LNA work well with various standards, while the folded-cascode structure with feedback resistance improves the overall performance of LNA.

TABLE I. OPERATING FREQUENCIES OF VARIOUS STADARDS

\begin{tabular}{|c|c|}
\hline Standard & Frequency $\mathbf{( M H z )}$ \\
\hline WCDMA & $2130-2145$ \\
\hline CDMA2000 & $2110-2125$ \\
\hline TD-SCDMA & $2010-2025$ \\
\hline Bluetooth & 2400 \\
\hline
\end{tabular}

\section{THEORY AND DESIGN}

\section{A. Current-controled Active Inductor}

As shown in Fig. 1, active inductors usually adopt the Gyrator-C structure [2-4], using CMOS transistors to achieve the spiral inductor function. The advantages of this structure include easy tuning of inductance with high quality factor $(Q)$ and occupying less chip area than conventional spiral inductors. According to Fig. 1 , the equivalent inductance $\left(l_{e q}\right)$ and the equivalent resistor $\left(r_{e q}\right)$ can be expressed as

$$
l_{e q}=\frac{g_{m 2} g_{m 3} c_{g s 2}+\omega^{2} c_{g s 2}^{2} c_{g s 3}\left(R g_{d s 3}+1\right)}{g_{m 2}^{2} g_{m 3} g_{m 1}+\omega^{2} g_{m 3} g_{m 1} c_{g s 2}^{2}},
$$

$$
r_{e q}=\frac{g_{m 2} g_{d s 3} g_{d s 1}+\omega^{2}\left(g_{m 3} c_{g s 2}^{2}-g_{m 2} c_{g s 2} c_{g s 3}\left(R g_{d s 3}+1\right)\right)}{g_{m 2}^{2} g_{m 3} g_{m 1}+\omega^{2} g_{m 3} g_{m 1} c_{g s 2}^{2}},
$$

where $g_{m 1}, g_{m 2}$ and $g_{m 3}$ are the transconductance of $\mathrm{M}_{1}, \mathrm{M}_{2}$ and $\mathrm{M}_{3}$, respectively, $C_{g s 2}$ and $C_{g s 3}$ are the parasitic gate-tosource capacitance of $\mathrm{M}_{2}$ and $\mathrm{M}_{3}$, respectively, $g_{d s 3}$ is the drain conductance of $\mathrm{M}_{3}, \omega$ is the operating frequency, and $R$ is the feedback resistance. According to (1) and (2), we can control the values of $l_{e q}$ and $r_{e q}$ by adjusting $g_{m 1}, g_{m 2}$ and $g_{m 3}$.

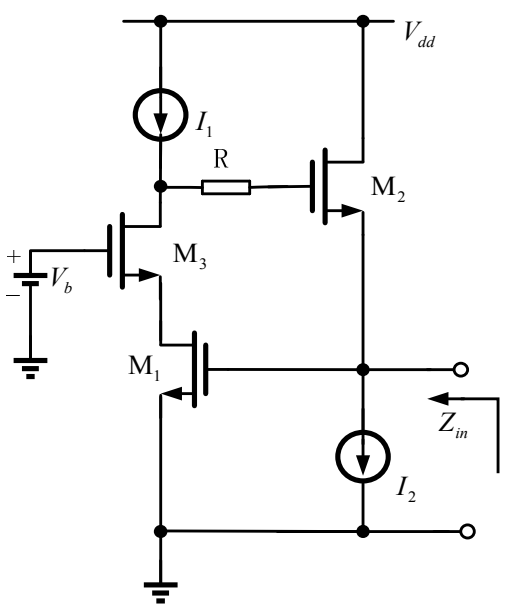

Figure 1. Schematic circuit diagram of active inductor 
The relation between the transconductance $\left(g_{m}\right)$ and the bias current $(I)$ of MOS transistor can be expressed as

$$
g_{m}=\frac{2 I}{V_{G S}-V_{T H}},
$$

where $V_{G S}$ is the gate-source voltage and $V_{T H}$ is the threshold voltage. According to (3), $g_{\mathrm{m}}$ can be controlled by the bias current to meet different communication standards. In the circuit shown in Fig. $1, g_{m 1}$ and $g_{m 3}$ change with $I_{1}$ while $g_{m 2}$ changes with $I_{2}$.

\section{B. Multi-standard LNA}

As shown in Fig. 2, the designed multi-standard LNA circuit topology adopts an inductor-degenerated common source structure [5-7], in which the proposed currentcontrolled active inductors are used for impedance matching with different standards.

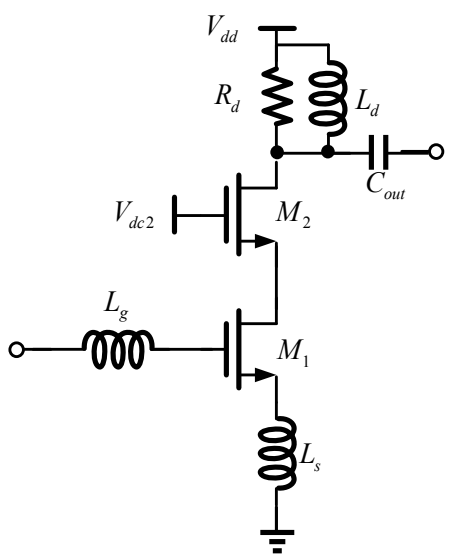

Figure 2. Multi-standard LNA based on active inductor

The input impedance $\left(Z_{i n}\right)$ of the LNA can be expressed as

$$
Z_{\text {in }} \approx s\left(L_{s}+L_{g}\right)+\frac{1}{s C_{g s 1}}+\frac{g_{m 1}}{C_{g s 1}} L_{s},
$$

where $C_{g s 1}$ is the parasitic capacitance of $\mathrm{M}_{1}$, and $s=j \omega$. To obtain a real input impedance of $50 \Omega$, the following equations must be satisfied:

$$
\begin{aligned}
& \omega\left(L_{\mathrm{g}}+L_{\mathrm{s}}\right)=\frac{1}{\omega C_{\mathrm{gs}}}, \\
& Z_{i n}=\frac{g_{m}}{C_{g s}} L_{s}=50 \Omega .
\end{aligned}
$$

The noise figure of the multi-standard LNA is

$$
N F \approx 1+\frac{1}{R_{s}}\left(\frac{\omega L_{g}}{Q_{L g}}+\frac{\omega L_{s}}{Q_{L s}}\right)+\gamma g_{d 0} R_{s} \frac{\omega C_{g s 1}}{g_{m 1}}+\frac{\left(\omega L_{s}\right)^{2}}{R_{s} R_{d}},
$$

where $R_{\mathrm{s}}$ is the source resistance (usually $50 \Omega$ ), $R_{d}$ is the load resistance, $g_{d 0}$ is the drain conductance of $\mathrm{M}_{1}$ at zero bias, $Q_{L g}$ and $Q_{L s}$ are quality factors of the inductors $L_{s}$ and $L_{g}$, respectively, and $\gamma$ is the transfer constant. The channel thermal noise of $\mathrm{M}_{1}$ and the thermal noise from load resistor $R_{d}$ are also considered.

Due to the use of $R_{d}$, the parasitic effects will make the output impedance $\left(Z_{\text {out }}\right)$ unstable at high frequencies. To solve this problem, $R C$ resonant network is adopted in the multi-standard LNA. $Z_{\text {out }}$ can be expressed as

$$
Z_{\text {out }}=\frac{1}{s C_{\text {out }}}+\frac{s R_{d} L_{d}}{R_{d}+s L_{d}},
$$

where $C_{\text {out }}$ is the load capacitor, and $L_{d}$ is the load inductor. When $s C_{\text {out }}$ and $s L_{d}$ are much larger than $1, Z_{\text {out }}$ is approximately equal to $R_{d}$, suggesting that the output impedance is stable (not changing with operating frequency).

The linearity of the designed LNA is proportional to the overdrive voltage of $\mathrm{M}_{1}$ when $\mathrm{M}_{1}$ and $\mathrm{M}_{2}$ are biased in the high saturation region and kept far from the triode region. The linearity represented by the input third-order intercept point $\left(I I P_{3}\right)$ is

$$
I I P_{3} \approx V_{G S}-V_{T H},
$$

where $V_{G S}$ is the gate-source voltage of $\mathrm{M}_{1}$ and $V_{T H}$ is the threshold voltage. According to (4), $L_{g}$ and $L_{s}$ should vary correspondingly with different standards. However, tests have demonstrated that even keeping $L_{s}$ as a constant, different standards can also be satisfied. The values of $L_{g}$ are listed in Table 2 for different standards.

TABLE II. ACTIVE INDUCOTOR FOR DIFFERENT STANDERS

\begin{tabular}{|c|c|}
\hline Standard & $\boldsymbol{L}_{\boldsymbol{g}}(\mathbf{n H})$ \\
\hline WCDMA & 27.27 \\
\hline CDMA2000 & 27.83 \\
\hline TD-SCDMA & 30.66 \\
\hline Bluetooth & 21.53 \\
\hline
\end{tabular}

III. SIMULATION RESUlTS

\section{A. Quality Factor of Active Inductor}

Fig. 3 shows the obtained $Q$ for the proposed active inductor in the frequency range of $1.5 \mathrm{GHz}$ to $2.8 \mathrm{GHz}$. A maximum $Q$ of 63.2 is reached at $2.1 \mathrm{GHz}$.

B. S Parameters of Multi-standard LNA

The power gain $\left(S_{21}\right)$ of the multi-standard LNA is shown in Fig. 4 for different standards. $S_{21}$ exceeds $11.6 \mathrm{~dB}$ 
at operating frequencies of all standards. The maximum gain is $16.14 \mathrm{~dB}$ for Bluetooth at $2.4 \mathrm{GHz}$.

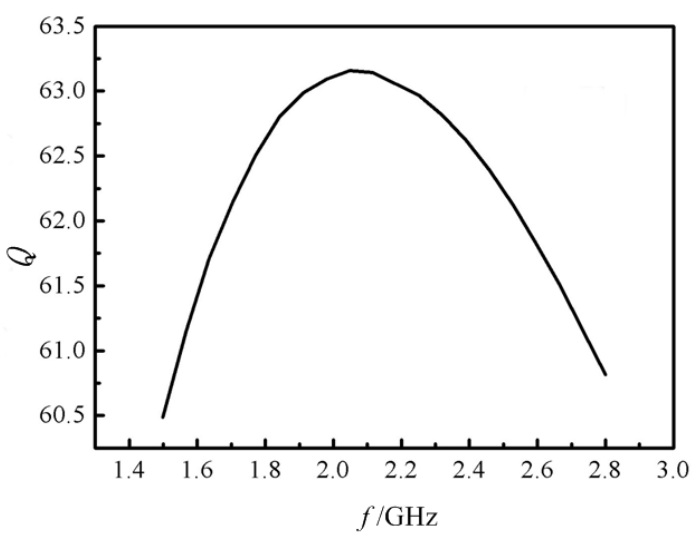

Figure 3. Quality factor of the active inductor

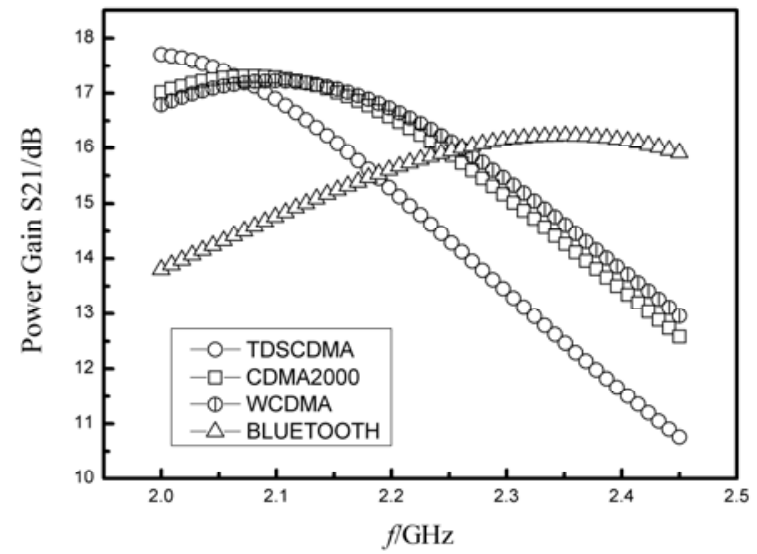

Figure 4. Power gain (S21)

The input matching $\left(S_{11}\right)$ is shown in Fig. 5. $S_{11}$ is well below $-54.01 \mathrm{~dB}$. The best matching $(-55.16 \mathrm{~dB})$ is obtained for TD-SCDMA at $2.017 \mathrm{GHz}$.

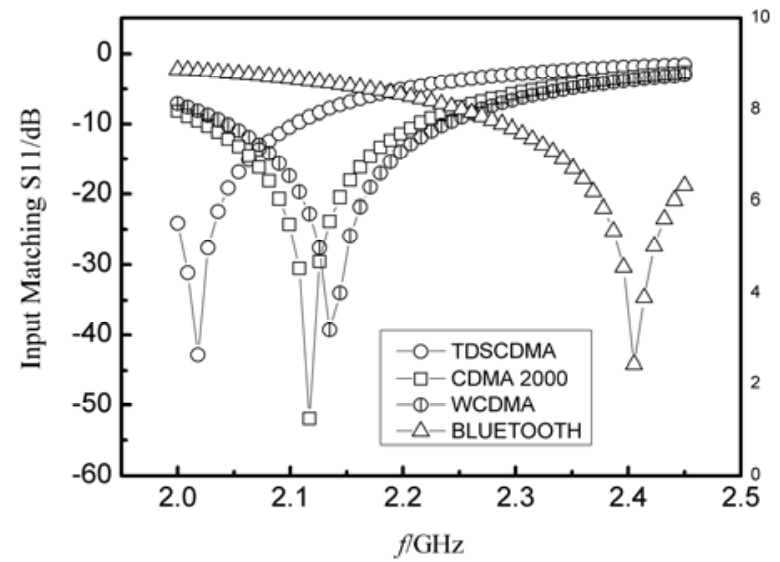

Figure 5. Input matching (S11)

Fig. 6 illustrates the changing tendency of the output matching $\left(S_{22}\right)$, which is well below $-26.78 \mathrm{~dB}$ for all desired frequencies. The minimal reflection at the load can ensure the efficient power transfer.

Fig. 7 shows the reverse isolation characteristics $\left(S_{12}\right)$. A minimum value of about $-47.18 \mathrm{~dB}$ in the entire frequency range offers good stability to the design LNA.

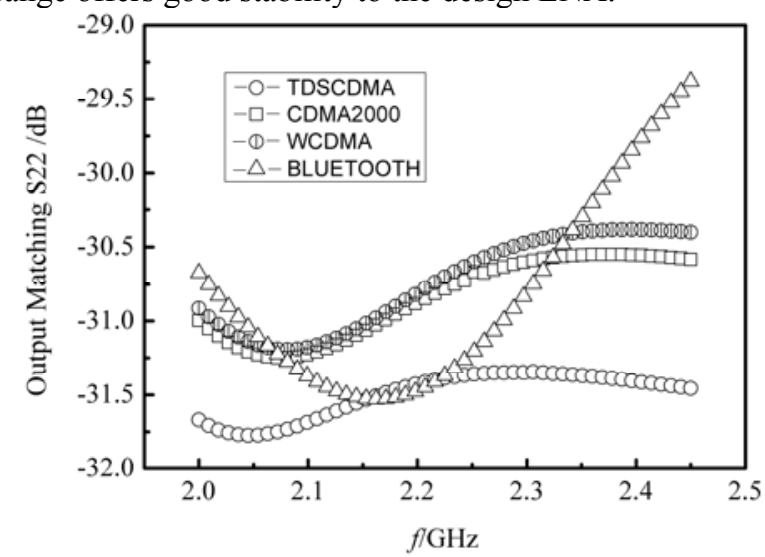

Figure 6. Output matching (S22)

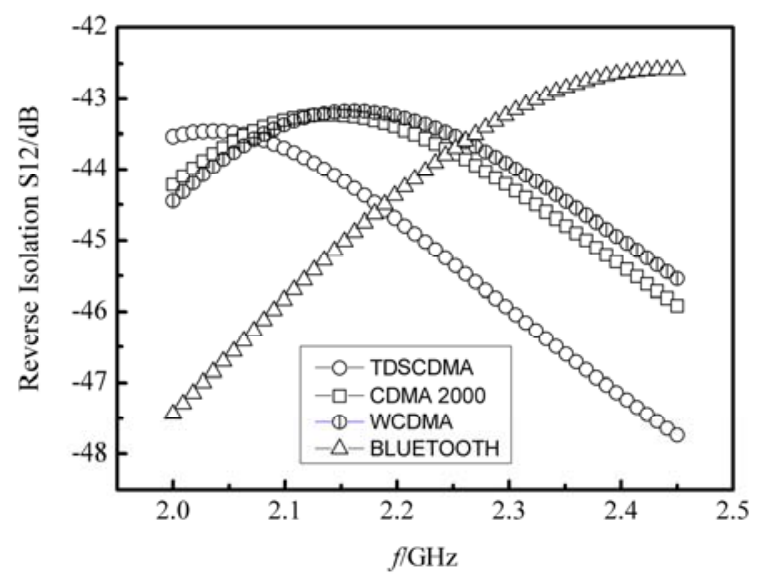

Figure 7. Reverse isolation (S12)

C. Noise figure of multi-standard LNA

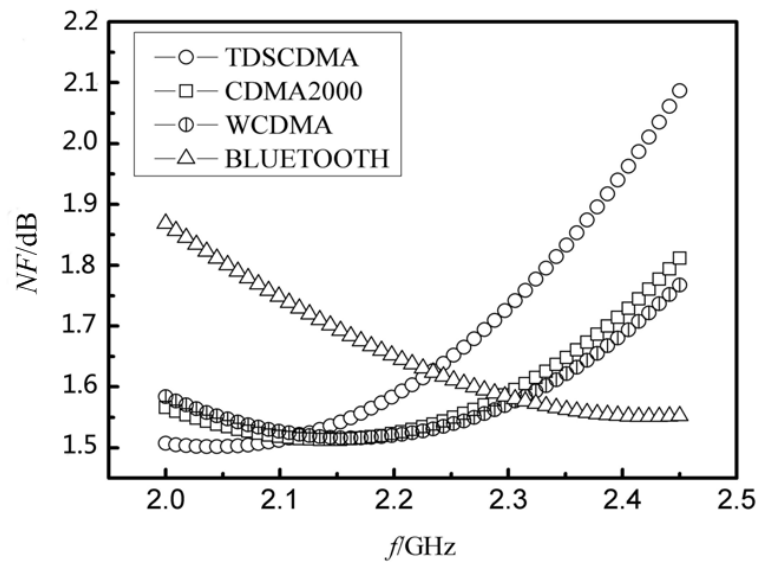

Figure 8. Noise figure of the multi-standard LNA 
Fig. 8 gives the noise figure of the designed LNA, which is smaller than $1.851 \mathrm{~dB}$ at required frequencies of the four standards. A minimum noise figure of $1.499 \mathrm{~dB}$ is obtained at $2.137 \mathrm{GHz}$ for WCMDA.

\section{SUMMARY}

A multi-standard LNA has been designed using $0.18 \mu \mathrm{m}$ CMOS technology based on the active inductor. The performance of the LNA is analyzed using Cadence. Simulation results of power gain, noise figure, input and output matching have demonstrated that the designed LNA meets the requirement of the four standards of CDMA2000, TD-SCDMA, WCDMA and Bluetooth. The tunable feature of the LNA is achieved by using the active inductor, which also helps to reduce the chip area.

\section{ACKNOWLEDGMENT}

This work is supported by National Natural Science Foundation of China (11074280), Natural Science Foundation of Jiangsu Province (BK2012110), Fundamental Research Funds for Central Universities of China (JUDCF12027, JUDCF12032), PAPD of Jiangsu Higher Education Institutions and the Summit of the Six Top Talents Program of Jiangsu Province (DZXX-053).

\section{REFERENCES}

[1] B.G. Perumana, Zhan, J.-H.C.and S.S Taylor., "Resistive-Feedback CMOS Low-Noise Amplifiers for Multiband Applications," IEEE Transactions on Microwave Theory and Techniques, vol.56, no.5, pp.1218-1225, May 2008.

[2] Cho Yong-Ho, Hong Song-Cheol and Kwon Young-Se, "A novel active inductor and its application to inductance-controlled oscillator," IEEE Transactions on Microwave Theory and Techniques, vol.45, no.8, pp.1208-1213, Aug 1997.

[3] J. Ngarmnil and C. Toumazou, "Micropower log-domain active inductor," Electronics Letters, vol.32, no.11, pp.953-955, 23 May 1996.

[4] H. Hayashi and M. Muraguchi, "A high-Q broad-band active inductor and its application to a low-loss analog phase shifter," IEEE Transactions on Microwave Theory and Techniques, vol.44, no.12, pp.2369-2374, Dec. 1996.

[5] F. Seguin , B. Godara and F. Alicalapa, "A gain-controllable wideband low-noise amplifier in low-cost $0.8-\mu \mathrm{m}$ Si BiCMOS technology," IEEE Transactions on Microwave Theory and Techniques, vol.52, no.1, pp. 154- 160, Jan. 2004.

[6] E.A. Sobhy, A.A. Helmy and S. Hoyos, "A 2.8-mW Sub-2-dB Noise-Figure Inductorless Wideband CMOS LNA Employing Multiple Feedback," IEEE Transactions on Microwave Theory and Techniques, vol.59, no.12, pp.3154-3161, Dec. 2011.

[7] F. Tzeng, A. Jahanian and P. Heydari, "A Multiband Inductor-Reuse CMOS Low-Noise Amplifier," IEEE Transactions on Circuits and Systems II: Express Briefs, vol.55, no.3, pp.209-213, March 2008. 